PROC. OF JSCE

No. 342, February 1984

\title{
DIFFUSION AND DISPERSION IN NARROW OPEN CHANNEL FLOW
}

\author{
By Norio HAYAKAWA*, Yusuke FUKUSHIMA** and Kenji SANJO***
}

\section{INTRODUCTION}

Longitudinal dispersion in the shear flow has been under extensive study ever since Taylor ${ }^{1)}$ first showed that, for a circular pipe, the shear dispersion far exceeds turbulent diffusion. Taylor's analysis was subsequently extended to the case of flow down an inclined plate by Elder ${ }^{2}$. Elder's result, supposedly to hold for open channel flow with a large width-to-depth ratio, turned out to give rather small dispersion coefficients for natural streams (Fischer $\left.{ }^{3}\right)$. For this phenomenon, Fischer ${ }^{4}$ gave an explanation that for natural streams the lateral velocity gradient predominates over the vertical one to become a determining factor of the longitudinal dispersion. Fischer showed a method for calculating the longitudinal dispersion due to the lateral velocity gradient giving a good agreement with both experimental and field data.

One then is left with a question what happens if width approaches depth. Granted that Fischer's method is applicable to natural streams with large width-to-depth ratios and that Elder's method for wide open channel flows with uniform depth. It is not infrequent that one encounters an open channel flow with a narrow width; e.g. irrigation channels. It appears that little work has been reported concerning the problem of this kind. The purpose of this paper is to answer this question both theoretically and experimentally. In the following a theoretical study of the longitudinal dispersion of a semi-elliptical channel flow

* Member of JSCE, Ph. D., Professor, Department of Civil Engineering, Technological University of Nagaoka

** Member of JSCE, Dr. of Engineering, Associate Professor, Department of Civil Engineering, Technological University of Nagaoka

*** Member of JSCE, Master of Engineering, Osaka Prefecture is first described. This is followed by a laboratory flume study with a width-to-depth ratio down to 0.5 . Combined results are to give the longitudinal dispersion coefficient of the open channel flow with a small width-to-depth ratio.

\section{DISPERSION IN A SEMI-ELLIPTICAL CHANNEL}

Longitudinal dispersion in a uniform, steady flow is caused by the interaction of the velocity gradient in the cross-section (the shear effect) and turbulent mixing within it: a phenomenon called as shear dispersion. Therefore, in Elder's method a wide open channel flow is represented by a flow with uniform vertical shear. Fischer's insight is to observe that in natural streams lateral shear is much more predominant over vertical one, thus representing them by a flow with only a lateral shear.

As is well known, isovels in the open channel flow has a semi-enclosed shape with a maximum velocity at around the center of the channel and shows decreasing velocity toward the bottom and the side walls. According to the basic concept of shear dispersion therefore, the major causes of dispersion are vertical shear and vertical turbulent diffusion around the center portion of the cross-section, whereas near the side walls they are the lateral shear and lateral turbulent diffusion. Generally at any point in the cross-section, shear dispersion is caused by the local maximum of velocity gradient and turbulent diffusion in that direction. Dispersion coefficient of an open channel flow should be calculable once the isovels and turbulent diffusion distribution are known in the cross-section. Calculation of dispersion coefficient for open channel flow with an arbitrary cross-sectional shape would be greatly simplified if the turbulent diffusion equation for a conservative tracer is written in terms of the orthogonal curvilinear coordinate system consisting of isovels, $\xi$-curves, and their orthogonal trajectories, $\eta$-curves, as follows: 


$$
\begin{aligned}
& \frac{\partial}{\partial t}\left(\bar{c}+c^{\prime}\right)+u^{\prime} \frac{\partial}{\partial x_{i}}\left(\bar{c}+c^{\prime}\right) \\
& \quad=\frac{1}{h_{\xi} h_{\eta}}\left[\frac{\partial}{\partial \xi}\left(\frac{h_{\xi}}{h_{\eta}} K_{\xi} \frac{\partial c^{\prime}}{\partial \xi}\right)+\frac{\partial}{\partial \eta}\left(\frac{h_{\eta}}{h_{\xi}} K_{\eta} \frac{\partial c^{\prime}}{\partial \eta}\right)\right]
\end{aligned}
$$

in which $\bar{c}$ is the cross-sectional mean concentration of a tracer, $c^{\prime}$ is a deviation therefrom, $t$ is time, $u^{\prime}$ is velocity deviation from cross-sectional mean, $x$ is a longitudinal coordinate moving with mean velocity averaged over a cross-section, $h_{\xi}$ and $h_{\eta}$ are scale factors of the $\xi$ and $\eta$ coordinates, and $K_{\xi}$ and $K_{\eta}$ are turbulent diffusion coefficients. Invoking the assumption following Taylor that $\bar{c}$ is a function only of $x$ and $c^{\prime}$ a function only of $\xi$ enables integration of Eq. (1) to obtain the following :

$$
c^{\prime}=f(\xi) \frac{\partial \bar{c}}{\partial x}
$$

where

$$
f(\xi)=\int_{\xi}\left[\left(K_{\xi} \int_{h_{\xi}} \frac{h_{\eta}}{h_{\xi}} d \eta\right)^{-1} \int_{\xi} \int_{\eta} u^{\prime} h_{\xi} h_{\eta} d \eta d \xi\right] d \xi
$$

The longitudinal dispersion coefficient is calculated as follows:

$$
D=\frac{-1}{A} \int_{A} u^{\prime} f d A
$$

It is easy to see that the general equations (2) and (3) reduce themselves to the Elder's model if $\xi$ and $\eta$ are set as $z$ (vertical axis) and $y$ (horizontal axis) respectively, and to the Fischer's model if $\xi$ and $\eta$ are set as $y$ and $z$ respectively.

To further the theoretical study, a good mathematical representation of $\xi-\eta$ coordinate system is necessary so that an integration of Eqs. (2) and (3) is tractable. At present, this is not possible for a rectangular shaped open channel flow. The purpose of this paper is to obtain a qualitative trend as the width-to-depth ratio of an open channel is reduced. Therefore a theoretical calculation is carried out for a semi-elliptic shaped open channel for which a simple rempresentation of velocity profile is assumed although it may turn out not to be realistic for wide range of the width-to-depth ratio. The elliptical coordinate system is chosen to be $\xi-\eta$ coordinates (Fig. 1) and it is assumed that the local velocity $u$ follows the logarithmic law with respect to $\xi$ coordinate:

$$
u=\frac{u_{*}}{\kappa} \ln \left(1-\frac{\xi}{\xi_{0}}\right)+\frac{u_{\max }}{\kappa}
$$

in which $u_{*}$ is a friction velocity, $\kappa$ is a constant, $u_{\max }$ is the maximum velocity of the cross-section and $\xi_{0}$, representing the bottom of the channel, is related to the width $B$ and depth $H$ as follows:

$$
\xi_{0}=\frac{1}{2} \ln \frac{B+2 H}{|B-2 H|}=\frac{1}{2} \ln \frac{A_{r}+2}{\left|A_{r}-2\right|}
$$

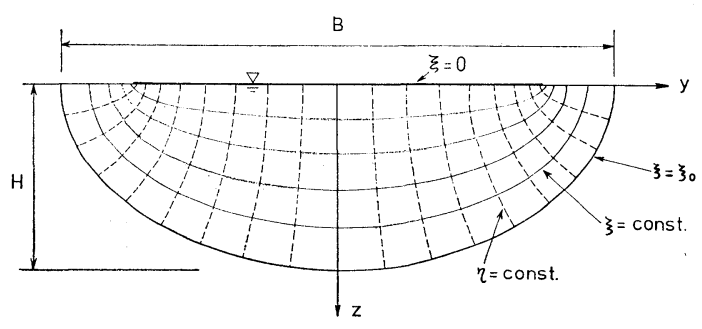

Fig. 1 Orthogonal coordinates of semi-elliptic channel.

in which $A_{r}=B / H$ is width-to-depth ratio. The expression (4) follows observed result of Chiu and Hsiung $^{5}$.

It should be noted here that adoption of elliptical coordinate system to represent isovels poses a serious difficulty as $A_{\boldsymbol{r}} \rightarrow 2$. For Eq. (5) indicates that $\xi_{0}$, thus in turn $u$, diverges under such condition. It is regrettable, therefore, that the present analysis is expected not to hold in the region around $A r=2$. However, at present no alternate approach which is mathematically tractable is not known and, as stated before, the present analysis is carried out in order to obtain a qualitative trend as $A_{r}$ is changed. Analytical result then is compared with experimental result and intuitive foresight.

Scaling factors $h_{\xi}$ and $h_{\eta}$ are given as follows:

$$
h_{\xi}=h_{\eta}=c \sqrt{\sinh ^{2} \xi+\sin ^{2} \eta}
$$

in which $c=\sqrt{(B / 2)^{2}-H^{2}}$.

Substituting Eqs. (4) and (6) into Eqs. (2) and (3), the longitudinal dispersion coefficient is obtained as follows:

$$
D=\frac{-c^{2} u *^{2}}{K_{\xi} \kappa \sinh \left(2 \xi_{0}\right)} F\left(\xi_{0}\right)
$$

where

$$
\begin{aligned}
F\left(\xi_{0}\right)= & \int_{0}^{\xi_{0}}\left[\int_{\xi} \int_{\xi}\left\{\ln \left(1-\frac{\xi}{\xi_{0}}\right)-u^{\prime \prime}\right\}\right. \\
& \cdot \cosh 2 \xi d \xi d \xi\left\{\ln \left(1-\frac{\xi}{\xi_{0}}\right)-u^{\prime \prime}\right\} \\
& \cdot \cosh 2 \xi] d \xi \ldots \ldots \ldots \ldots \ldots \ldots \ldots \ldots \ldots \ldots \ldots \ldots \ldots
\end{aligned}
$$

in which $u^{\prime \prime}$ is defined as follows:

$$
u^{\prime \prime}=\frac{2}{\sinh \left(2 \xi_{0}\right)} \int_{0}^{\xi_{0}} \ln \left(1-\frac{\xi}{\xi_{0}}\right) \cosh 2 \xi d \xi
$$

Turbulent diffusion coefficient $K_{\xi}$ is expressed as follows:

$$
K_{\xi}=(1 / 6) \kappa R u_{*}
$$

in which $R$ is a hydraulic radius calculated as: 


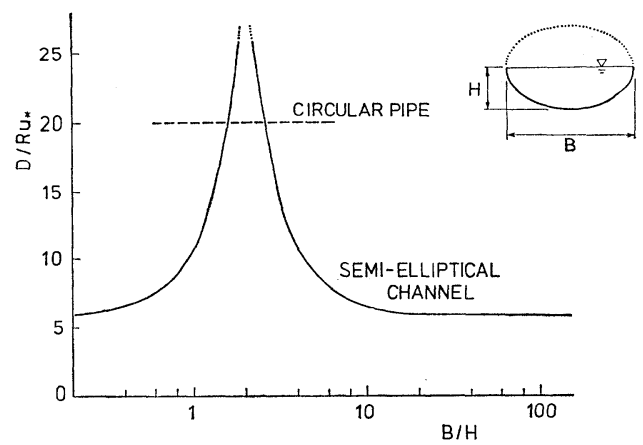

Fig. 2 Longitudinal dispersion coefficient of semi-elliptic channel.

$$
R=\frac{\pi c \sinh \left(2 \xi_{0}\right)}{8}\left[\int_{0}^{\pi / 2} \sqrt{\cosh ^{2} \xi_{0}-\cos ^{2} \eta} d \eta\right]^{-1}
$$

Expression (8) is obtained assuming Reynolds analogy and averaging over $\xi$ coordinate.

Eq. (7) is then written in non-dimensional form as

$$
\begin{aligned}
\frac{D}{R u *}= & -\frac{384}{\pi^{2} \kappa^{3} \sinh ^{3}\left(2 \xi_{0}\right)} \\
& \cdot\left[\int_{0}^{\pi / 2} \sqrt{\cosh ^{2} \xi_{0}-\cos ^{2} \eta} d \eta\right]^{2} F\left(\xi_{0}\right)
\end{aligned}
$$

In Eq. (10), the left hand side is a non-dimensional dispersion coefficient and the right hand side is a function only of $\xi_{0}$, that is, in turn, $A \boldsymbol{r}$. Integration of $F\left(\xi_{0}\right)$ is carried out by reducing it to a system of ordinary differential equations and calculated value of $D / R u_{*}$ assuming $\kappa=0.39$ is shown in Fig. 2.

In Fig. 2, non-dimensional dispersion coefficient $D /(R u *)$ is found to be symmetrical about $A$ r $=2$ and approaches the Elder's value as both $A_{r} \rightarrow \infty$ and $A_{\boldsymbol{r}} \rightarrow 0$. At $A_{\boldsymbol{r}}=2$, the calculated dispersion coefficient diverges because of unrealistic behavior of the velocity distribution adopted, i.e. Eq. (4). Theoretically, $D$ should be equal to that of a circular pipe at $A \boldsymbol{r}=2$ if a good velocity distribution is adopted. Therefore, it is concluded herewith that the theoretical dispersion coefficient, starting with the value for the circular pipe at $A_{\boldsymbol{r}}=2$, should decrease to the Elder's value as either $A_{r \rightarrow \infty}$ or $A_{r} \rightarrow 0$ in much the same way as is given in Fig. 2. In the real turbulent flow, however, $D$ could be much larger than is predicted by the theory because of the existence of the secondary flow, which has been totally ignored in the present analysis.

\section{EXPERIMENTAL STUDY}

\section{(1) Apparatus and Procedure}

Experimental study of diffusion and dispersion is carried out with a laboratory flume $10.5 \mathrm{~m}$ long, $9.9 \mathrm{~cm}$ wide and $30 \mathrm{~cm}$ high. The flume is built with wood and its inside is painted black with white painted markings. Table 1 gives the hydraulic parameters of all the experimental runs. Runs no. 5 and 6 are carried out to obtain the longitudinal dispersion coefficient only.

Table 1 Hydraulic parameters.

\begin{tabular}{c|c|c|c|c|c|c}
\hline Run & $\begin{array}{c}H \\
\text { Depth } \\
(\mathrm{cm})\end{array}$ & $\begin{array}{c}A_{r} \\
\text { Width- } \\
\text { to-depth } \\
\text { ratio }\end{array}$ & $\begin{array}{c}\bar{u} \\
\text { Mean } \\
\text { velocity } \\
(\mathrm{cm} / \mathrm{s})\end{array}$ & $\begin{array}{c}u_{*} \\
\text { Friction } \\
\text { velocity } \\
(\mathrm{cm} / \mathrm{s})\end{array}$ & $\begin{array}{c}R \\
\text { Hydrau- } \\
\text { lic radius } \\
(\mathrm{cm})\end{array}$ & $\begin{array}{c}R e \\
\text { Reynolds } \\
\text { number }\end{array}$ \\
\hline 1 & 5.0 & 2 & 20.0 & 1.54 & 2.49 & 5000 \\
2 & 10.0 & 1 & 20.9 & 1.44 & 3.31 & 6600 \\
3 & 15.0 & 0.67 & 18.6 & 1.41 & 3.72 & 7400 \\
4 & 20.0 & 0.5 & 19.7 & 1.39 & 3.97 & 7900 \\
5 & 3.33 & 3 & 19.3 & 1.55 & 1.99 & 3800 \\
6 & 2.5 & 4 & 18.8 & 1.61 & 1.66 & 3100 \\
\hline
\end{tabular}

Velocity distribution is obtained with use of a small propellar current meter ( $3 \mathrm{~mm}$ diameter) supplemented with occasional usage of a Pitot tube. Diffusion and dispersion at the water surface is measured by following the movement of a confetti ( $2 \mathrm{~mm}$ diameter) by a video camera. A confetti was dropped at $2 \mathrm{~m}$ from the upstream end and its path is followed for $3 \mathrm{~m}$ to ensure that dispersion-dominant region is included. For each run, 100 such experiments are performed. For dispersion study a slug of $\mathrm{NaCl}$ solution is released at the upstream end and salt concentration is measured at $7.5,8.5$ and $9.5 \mathrm{~m}$ downstream with conductivity probes placed at the midsection. Measured concentration at these points are thought to represent cross-sectional average values from viewpoint of the lateral diffusion data.

\section{(2) Velocity Distribution}

Fig. 3 shows velocity distributions, i.e. isovels, for four runs. Velocity data are obtained at 99 grid points shown in Fig. 3. The isovels are observed to be of semi-enclosed shape as are generally the case. Moreover, following tendencies are observed: (a) The maximum velocity appears at the center line and its point tends to descend as the width-to-depth ratio is reduced, (b) isovels tend to converge toward the midwidth around the water surface and their shape approaches ellipses except for the proximity of side walls, and (c) around the corners and water surface, velocity tends to decrease, suggesting 

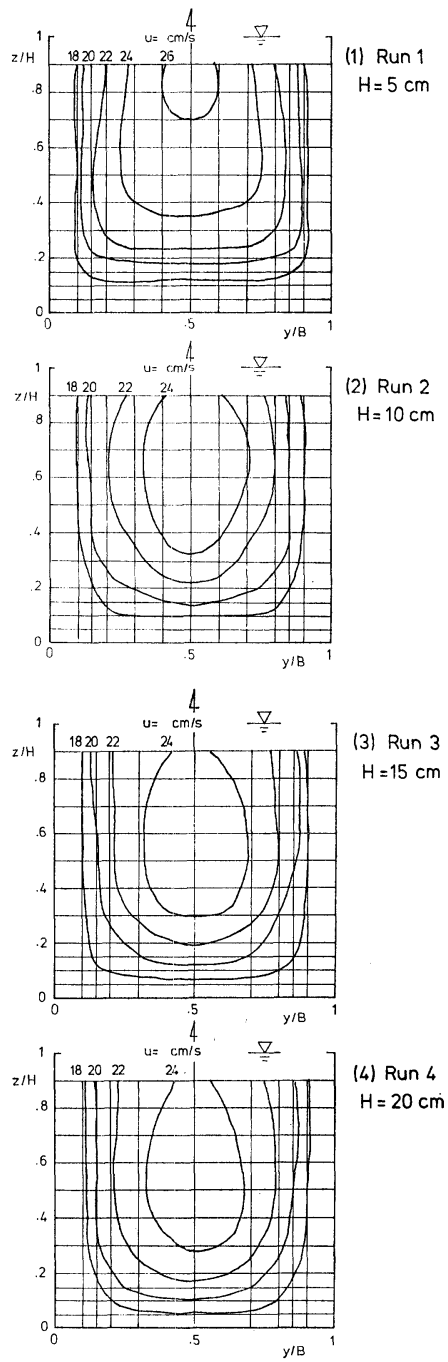

Fig. 3 Measured velocity distribution (isovels) in the flume.

existence of the secondary flow.

These measured data can be used to obtain a good mathematical representation of the velocity profile such as shown by Chiu and Hsiung ${ }^{5)}$. Still it is not possible to calculate the dispersion coefficient with such a profile and a further effort is not presented in this paper. It should be pointed out, however, that the measured isovels look somewhat close to ellipses when the widthto-depth ratio is large (Run 1 and 2), whereas they depart from the assumption adopted in the theoretical study in that the maximum velocity is obtained underneath the water surface.

\section{(3) Confetti Dispersion on the Water Surface}

Figs. 4 and 5 are, respectively, lateral and longitudinal dispersions of confetti with time.

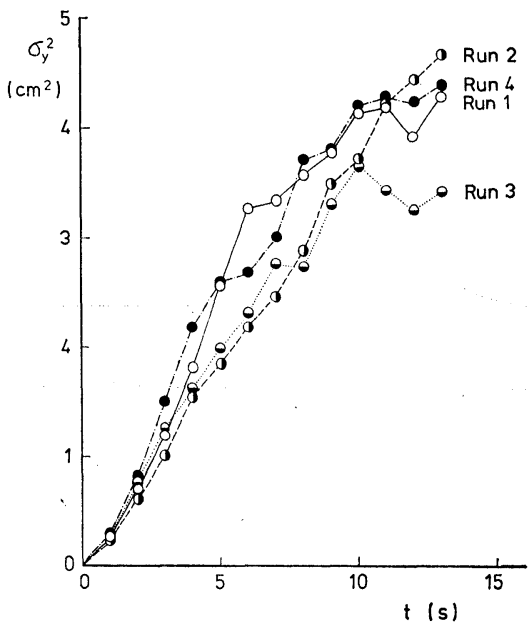

Fig. 4 Lateral dispersion of confetti with time.

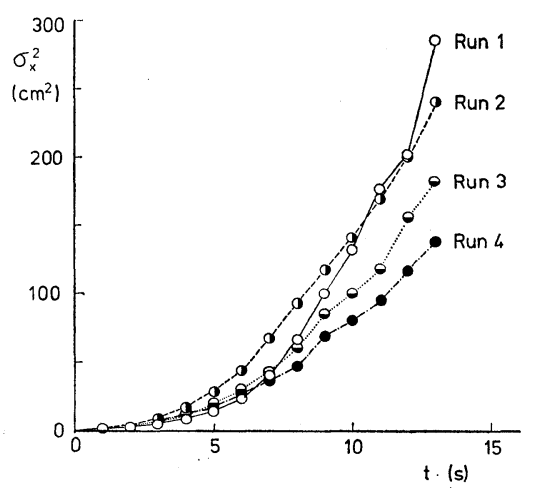

Fig. 5 Longitudinal dispersion of confetti with time.

The lateral dispersion tends to level off for large time, showing the influence of the side walls, whereas the longitudinal dispersion tends to increase parabolically with time. According to Fischer ${ }^{4}$, dispersion of tracer particles is divided into two periods: (a) initial convection period during which the movement of tracer particles is still dependent on their initial convective velocity; and (b) the diffusive period, during which the movement of tracer particles is described by the longitudinal dispersion coefficient. Fischer found, in his study with three-dimensional flume, the diffusive period to start at the time six times the Lagrangian time scale, thus $1.8 l^{2} /\left(R u_{*}\right)$, in which $l$ is the distance between the maximum velocity point and the side wall. In the present study this time is calculated to be 8 to 11.7 seconds. Fischer's result, however, was obtained with a wide 'three-dimensional' flow and it is open to question if his result applies to the present study. In experiment it is observed that 9 seconds after the 
Table 2 Diffusion and dispersion coefficient.

\begin{tabular}{|c|c|c|c|c|c|c|c|c|}
\hline \multirow{2}{*}{ Run. no. } & \multicolumn{2}{|c|}{$\begin{array}{l}\text { Longitudinal turbulent } \\
\text { diffusion coefficient }\end{array}$} & \multicolumn{2}{|c|}{$\begin{array}{c}\text { Lateral turbulent } \\
\text { diffusion coefficient }\end{array}$} & \multicolumn{2}{|c|}{$\begin{array}{c}\text { Surface dispersion } \\
\text { coefficient }\end{array}$} & \multicolumn{2}{|c|}{$\begin{array}{c}\text { Longitudinal dispersion } \\
\text { coefficient }\end{array}$} \\
\hline & $K_{x}\left(\mathrm{~cm}^{2} / \mathrm{s}\right)$ & $K_{x} /\left(R u_{*}\right)$ & $K_{y}\left(\mathrm{~cm}^{2} / \mathrm{s}\right)$ & $K_{y} /\left(R u_{*}\right)$ & $D_{s}\left(\mathrm{~cm}^{2} / \mathrm{s}\right)$ & $D_{s} /\left(R u_{*}\right)$ & $D\left(\mathrm{~cm}^{2} / \mathrm{s}\right)$ & $D /\left(R u_{*}\right)$ \\
\hline 1 & 0.57 & 0.15 & 0.30 & 0.089 & 24.2 & 6.45 & 53 & 14.1 \\
\hline 2 & 0.73 & 0.15 & 0.20 & 0.042 & 16.4 & 3.45 & 65 & 13.7 \\
\hline 3 & 0.67 & 0.13 & 0.20 & 0.038 & 13.8 & 2.64 & 60 & 11.5 \\
\hline 4 & 0.71 & 0.13 & 0.26 & 0.046 & 9.9 & 1.79 & 60 & 10.8 \\
\hline 5 & - & - & - & - & - & - & 47 & 15.2 \\
\hline 6 & - & - & - & - & - & - & 39 & 14.6 \\
\hline
\end{tabular}

release confetti are uniformly distributed over the channel width. Therefore, the longitudinal turbulent diffusion coefficient, $K_{x}$, is calculated using the confetti dispersion data from 1 to 6 seconds after release and finding the temporal growth rate at 1 second. The lateral turbulent diffusion coefficient, $K_{y}$, is similarly calculated using the confetti dispersion data from 1 to 6 seconds after release. For the period from 10 to 13 seconds, the 'surface' longitudinal dispersion coefficient, $D_{s}$, is then calculated using the longitudinal confetti dispersion data. These values are all listed in Table 2.

Measured longitudinal diffusion coefficients divided by the product of hydraulic radius and friction velocity, $K_{x} /\left(R u_{*}\right)$, are about 0.15 and insensitive to change in the width-to-depth ratio, $B / H$. They are much higher than the value, 0.068 , suggested by Elder ${ }^{2)}$ who assumed isotropy in diffusivity and Reynolds analogy. Engelund ${ }^{6)}$, who did the study almost identical to the authors but with a wide open channel, reported $K_{x} /\left(R u_{*}\right)$ of 0.51 and 0.90 . Richardson and Miller ${ }^{7}$ carried out a tracer diffusion study with a narrow open channel $(B / H=4.7)$ which had enhanced bottom roughness. They measured lateral diffusion coefficient and, claiming that the longitudinal diffusion is three times the lateral, obtained $K_{x} /\left(R u_{*}\right)$ in the range of $0.42-0.82$. There is not enough data available to further discuss this matter and it is only noted herewith that the value of this study is probably to the lower side.

Lateral diffusion coefficient has a marked tendency of decreasing as the width-to-depth ratio decreases. Fig. 6 is a plotting of $K_{y} /\left(R u_{*}\right)$ versus $B / H$ together with the data of some of the earlier published works related to the smooth open channel flow (Data are taken from the paper by Lau and Krishnappen ${ }^{8)}$ ). Fig. 6 shows that the data of this study are smoothly connected to those of the earlier works and that $K_{y} /\left(R u_{*}\right)$ tends to decrease as $B / H$ decreases. It is possible to theorize this decreasing tendency by assuming that the lateral mixing at the water surface when $B / H$ is small is governed by the side wall alone because the channel bottom is smooth in this study. Fig. 7 shows that the surface velocity

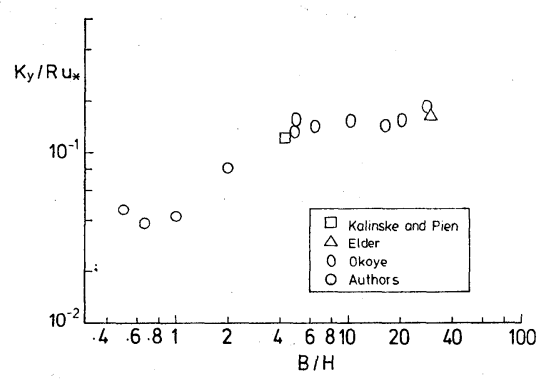

Fig. 6 Lateral turbulent diffusion coefficient versus width-to-depth ratio.

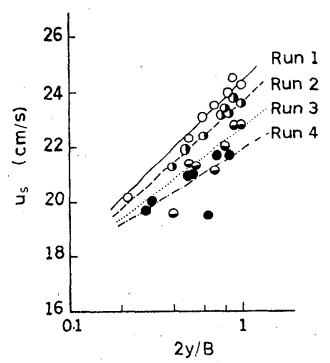

Fig. 7 Surface velocity distribution ( $y$ : distance from the side wall).

distribution does indeed follow the logarithmic law with distance $y$ measured from the side wall which can be written as

$$
u=u_{* s} \frac{1}{\kappa} \ln (2 y / B)
$$

in which $u_{* s}$ is the friction velocity pertaining to the side wall. From Fig. $7 u_{* s}$ is obtained assuming $\kappa=0.39$ and calculated values of $2 . K_{y} /$ $\left(u_{* s} B\right)$ are listed in Table 3 . It is observed that the caclulated values are close to the theoretical value of 0.067 , which can be obtained assuming Reynolds analogy, Eq. (11) and linear change in the shear stress and, finally, averaging over the width.

The surface dispersion coefficient and its nondimensional form, $D_{s} /(R u *)$, also exhibit a marked tendency to decrease as the width-to-depth ratio is decreased. As before, assuming Reynolds 
Table 3 Surface friction velocity and diffusion/ dispersion coefficient.

\begin{tabular}{c|c|c|c}
\hline Run no. & $\begin{array}{c}\text { Surface friction } \\
\text { velocity } \\
u_{* s}\left(\mathrm{~cm}^{2} / \mathrm{s}\right)\end{array}$ & $2 K_{y} /\left(B u_{* s}\right)$ & $2 D_{s} /\left(B u_{* s}\right)$ \\
\hline 1 & 1.03 & 0.059 & 4.74 \\
2 & 0.85 & 0.047 & 3.89 \\
3 & 0.80 & 0.051 & 3.48 \\
4 & 0.63 & 0.083 & 3.17 \\
\hline
\end{tabular}

analogy and Eq. (11) it is possible to conceive 'surface' shear dispersion with the predicted value for $2 D_{s} /\left(u_{* s} B\right)$ of 5.86 , numerically identical to Elder's model. Table 3 lists calculated values of $2 D_{\boldsymbol{s}} /\left(u_{* s} B\right)$, which are found to be more uniform than $D_{s} /(R u *)$, but a little lower than the predicted still showing decreasing tendency as $B / H$ decreases.

\section{(4) Longitudinal Dispersion}

The longitudinal dispersion coefficients for each run were obtained by the routing procedure, i.e. finding the dispersion coefficient that gives best prediction of the downstream concentration profiles out of a given upstream concentration profile. Calculated longitudinal dispersion coefficients $D$ and their non-dimensional forms $D /\left(R u_{*}\right)$ are given in Table 2. Fig. 8 shows the plotting of $D /\left(R u_{*}\right)$ versus $B / H$ together with the theoretical prediction for a semi-elliptic channel and some of the flume data for small $B / H$ values. Of earlier works, Richardson and Miller's data are obtained with rough bottom and some of Fischer's data with tick marks are obtained with flow over sand dunes. Therefore, they cannot be really compared with the present study but show the influence of enhanced roughness on the dispersion in flumes. Data of Iwasa and $\mathrm{Aya}^{9)}$ and Michiue $e$ t $a l^{10)}$ tend to give larger $D /(R u *)$ values than other flume data. The reason for this is not clear and it is only suspected that their flumes somehow have seen development of a secondary flow.

It is observed in Fig. 8 that authors' data form a smooth lower bound for the flume data and an addition of roughness, possibly including existence of secondary flow, would raise the dispersion coefficient.

The theoretical prediction for width-to-depth ratio larger than 4 gives smaller $D /\left(R u_{*}\right)$ values than experimental data. This is due to the fact that the theory assumes flow over smooth bed and no secondary flow whereas the experimental values are enhanced by these factors. This is especially the case for the data of Richardson and Miller. For the width-to-depth ratio of around 2.0, the theory breaks down owing to unrealistic behavior of the velocity profile as

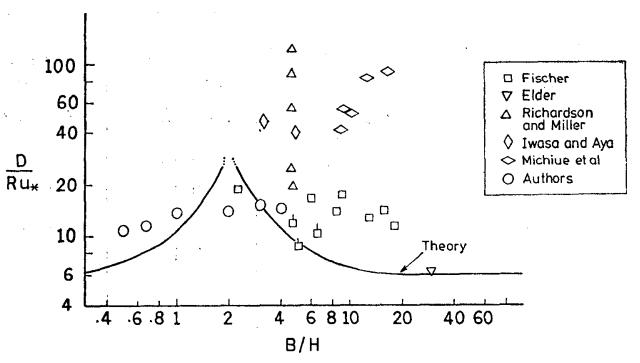

Fig. 8 Longitudinal dispersion coefficient versus width-to-depth ratio (tick marks on Fischer's data indicates runs with sand dunes).

discussed previously. If a more reasonable velocity profile is adopted, the theoretical value of $D /\left(R u_{*}\right)$ should coincide with Taylor's value of 20.2 and experimental data of this study tend to support this proposition.

\section{CONCLUSION}

Longitudinal dispersion of narrow open channel flow is studied both theoretically and experimentally. In the course of study, some knowledge on turbulent diffusion is also gained. Followings are the conclusions obtained in this study.

(1) A theoretical study on the shear dispersion in a semi-elliptic open channel shows that non-dimensional longitudinal dispersion coefficient $D /\left(R u_{*}\right)$ plotted against width-to-depth ratio $A_{r}$ is symmetrical about $A_{r}=2$ and approaches the Elder's value of 5.86 as both $A_{r \rightarrow \infty}$ and $A_{\boldsymbol{r}} \rightarrow 0$. At $A_{\boldsymbol{r}}=2$, the calculated dispersion coefficient diverges because of assumed velocity profile. If a more realistic velocity profile is adopted $D /\left(R u_{*}\right)$ should attain the value for a circular pipe at $A_{r}=2$. For other $A_{r}$ values, $D /$ $\left(R u_{*}\right)$ should behave in much the same way as is predicted in this study.

(2) Longitudinal and lateral dispersions of confetti on the water surface are measured in a laboratory flume for a range of $A_{r} 0.5$ to 2 and longitudinal and lateral turbulent diffusion coefficients, $K_{x}$ and $K_{y}$ respectively, as well as 'surface' longitudinal dispersion coefficient $D_{s}$ are obtained. Calculated values of $K_{y} /(R u *)$ are about 0.15 and insensitive to change in $A_{r}$, whereas $K_{y} /\left(R u_{*}\right)$ values tend to decrease as $A_{r}$ decreases. This tendency is attributed to the governing role of side walls with the result that $2 K_{y} /\left(u_{* s} B\right)$, in which $B$ is channel width and $u_{* s}$ is friction velocity pertaining to the side wall, is close to the theoretical value of $0.068 . D_{s} /$ $\left(R u_{*}\right)$ values also tend to decrease with decreasing $A$ r. A similar consideration as $K_{y}$ that the 
side wall effect determines $D_{\boldsymbol{s}}$ has met with only a limited success.

(3) Longitudinal dispersion coefficients are measured in a laboratory flume with $A_{\boldsymbol{r}}$ in the range of 0.5 to 4 . Comparison of measured longitudinal dispersion coefficients with earlier works and theoretical prediction (Fig. 8) shows that the data of the present study form a smooth lower bound for the flume data and an addition of roughness, possibly including existence of secondary flow, would raise the dispersion coefficient. For small values of width-to-depth ratio, a good correspondence of experimental data and theoretical prediction is observed.

\section{REFERENCES}

1) Taylor, G. I.: The dispersion of matter in turbulent flow through a pipe, Proc. Royal Soc. London, Vol. A223, pp. 446 468, 1954.

2) Elder, J. W.: The dispersion of marked fluid in turbulent shear flow, J. Fluid Mech., Vol. 5, pp. 544 560, 1959.

3) Fischer, H. B.: Longitudinal dispersion and turbulent mixing in open channel flow, Annual Review of Fluid Mech., Vol. 5, pp. 59 78, 1973.
4) Fischer, H. B.: The mechanics of dispersion in natural stream, J. Hyd. Div., Proc. ASCE, Vol. 93, HY6, pp. 187 216, 1967.

5) Chiu, Chao-Lin and D. E. Hsiung: Secondary flow, shear stress and sediment transport, J. Hyd. Div., Proc. ASCE, Vol. 107, HY7, pp. 879 898, 1981.

6) Engelund, F.: Dispersion of floating particles in uniform channel flow, J. Hyd. Div., Proc. ASCE, Vol. 95, HY4, pp. 1149 1162, 1969.

7) Richardson, E. V. and A. C. Miller: Diffusion and dispersion in open channel flow, J. Hyd. Div., Proc. ASCE, Vol. 100, HY1, pp. 159 171, 1974.

8) Lau, Y. L. and B. G. Krishnappen: Transverse dispersion in rectangular channels, J. Hyd. Div., Proc. ASCE, Vol. 103, HY10, pp. 1173 1189, 1977.

9) Iwasa, Y. and S. Aya: Dispersion coefficient of natural streams, Proc. 3rd Int'1 Symp. on Stochastic Hydraulics, pp. 527 550, 1980.

10) Michiue, M., Y. Muramoto and M. Itami: The role of convective period in the longitudinal dispersion (in Japanese), Proc. 22nd Japanese Conf. on Hydraulics, pp. 125 138, 1978.

(Received July 27, 1982) 